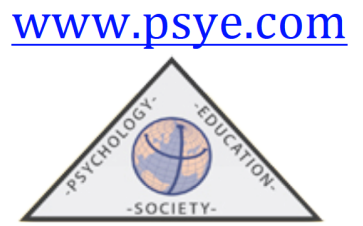

Psychology, Society, \& Education, 2019. Vol. 11(2), pp. 179-192

ISSN 2171-2085 (print) / ISSN 1989-709X (online)

Doi 10.25115/psye.v10i1.1927

\title{
Resultados diferenciales de la aplicación de dos programas en competencias emocionales en contexto escolar
}

\section{Lidia COBOS-SÁNCHEZ ${ }^{1}$, Juan M. FLUJAS-CONTRERAS ${ }^{2}$ e Inmaculada GÓMEZ ${ }^{3}$}

\author{
${ }^{1}$ (I.E.S. Sol de Portocarrero, Delegación de Educación, Junta de Andalucía, España; \\ y Centro de Investigación en Salud, Almería, España) \\ ${ }^{2}$ (Departamento de Psicología, Universidad de Almería, España; \\ y Centro de Investigación en Salud, Almería, España) \\ ${ }_{3}^{3}$ (Departamento de Psicología, Universidad de Almería, España; \\ y Centro de Investigación en Salud, Almería, España)
}

(Recibido, el 7 de Noviembre de 2018; Aceptado el 22 de Enero de 2019)

\begin{abstract}
RESUMEN: El objetivo del presente estudio es analizar los efectos de dos programas breves de intervención en inteligencia emocional; uno de ellos basado en el modelo de habilidades de inteligencia emocional, el programa INTEMO, y el otro un protocolo de intervención para el fomento de la flexibilidad psicológica con estrategias de mindfulness. Ambos protocolos se aplicaron a lo largo de cinco sesiones, cada una con una duración de una hora y con frecuencia semanal. Se sigue un diseño cuasi-experimental pretest-posttest con dos grupos independientes de medidas repetidas; la muestra inicial es de 215 sujetos, finalmente participaron 120 adolescentes españoles $(54.2 \%$ varones, con edad media de 12 años), procedentes de un centro de educación secundaria durante dos meses. Los resultados muestran diferencias entre los grupos en supresión emocional, regulación emocional e inteligencia emocional percibida. Igualmente se hallan conexiones entre inteligencia emocional y habilidades en mindfulness. El programa INTEMO obtiene puntuaciones significativamente diferentes y mayores en supresión emocional, regulación emocional e inteligencia emocional percibida. En el análisis de regresión se han hallado factores que parecen estar a la base del desarrollo de inteligencia emocional y mindfulness, encontrando conexión entre ambas.
\end{abstract}

Palabras clave: Inteligencia emocional, competencias emocionales, flexibilidad psicológica, mindfulness, adolescentes.

\section{Differential results of the application of two programs in emotional competences in school context}

\begin{abstract}
This study aims to analyse the effects of two briefs emotional intelligence (EI) program one based on the ability model, INTEMO program, and other based on psychological flexibility protocol with mindfulness strategies, along 5 sessions with 1-hour each. A pretest-posttest quasiexperimental design with two treatments groups; The initial sample was composed by 215 subjects, finally 120 Spanish adolescents (54.2\% male, mean age 12 years old) from a compulsory secondary education centre were involved in the study during two months. Results show differences in emotional suppression, emotional regulation and perceived emotional intelligence. Similarity, emotional
\end{abstract}


intelligence and mindfulness abilities has been related. INTEMO program obtain high and significant different punctuation in emotional suppression, emotional regulation and perceived emotional intelligence. In regression analysis some factors were found that can be in the base of the development of emotional intelligence and mindfulness, both are related.

Keywords: Emotional intelligence, emotional competences, psychological flexibility, mindfulness, adolescents.

Correspondencia: Inmaculada Gómez Becerra, Departamento Psicología. Escuela Superior de Ingeniería. Universidad de Almería. La Cañada de San Urbano, s/n. 04120, Almería (Spain). E-mail: igomez@ual.es

\section{Introducción}

La inteligencia emocional (IE) difiere en su definición y componentes en función del modelo explicativo; sin embargo, dos modelos han mostrado mayor aceptación y rigor científico (Trujillo Flores \& Rivas Tovar, 2005). Por un lado, el modelo de habilidad entiende la IE como un conjunto de destrezas para el procesamiento de la información de las emociones. Por otro lado, para el modelo mixto la IE es un conjunto de rasgos de personalidad y de competencias socio-emocionales (véase en la Figura 1 un esquema de los modelos más representativos) (García-Fernández \& Giménez-Mas, 2010).

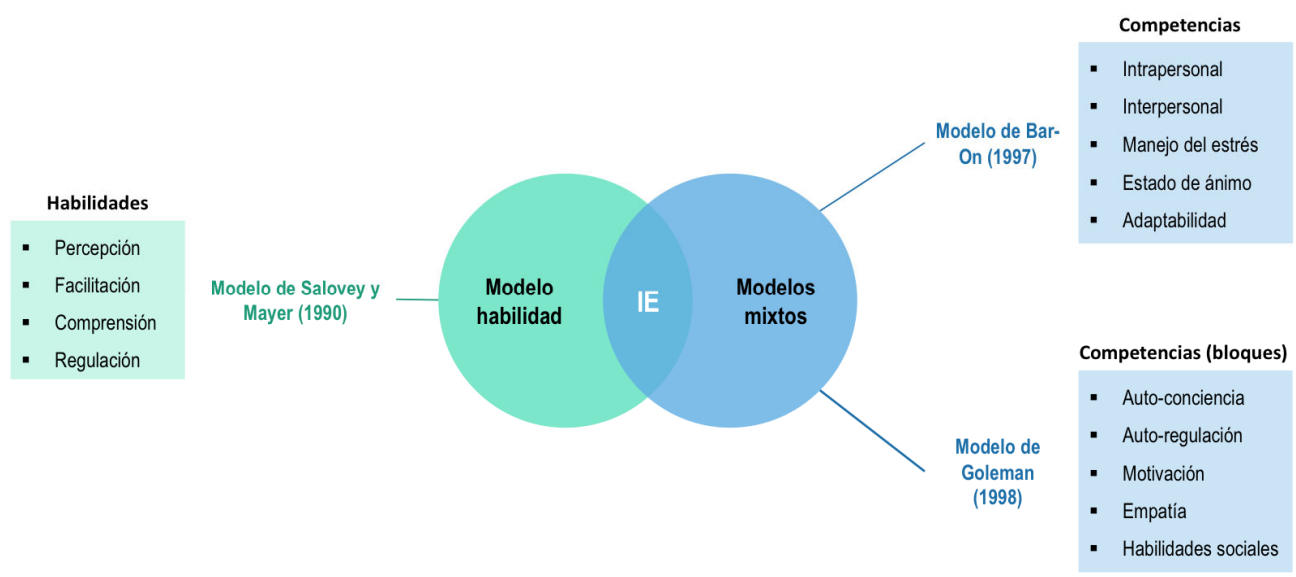

Figura 1. Principales modelos teóricos de la IE

Dada la importancia y el papel de la IE en el desarrollo y mantenimiento de ciertos problemas en la infancia y adolescencia, se han creado una serie de programas de educación emocional. En una revisión sistemática de programas de intervención en IE (Cobos, FlujasContreras, y Gómez, 2015) en España en el ámbito de la educación encontramos:

Por un lado, desde el modelo mixto de IE, el programa "Aprender a Ser Persona y a Convivir" (Trianes y Fernández-Figares, 2001) que ha mostrado mejoras en el clima social del aula (Carrasco, Alarcón, y Trianes, 2015); el programa "Aprender a Convivir para educación infantil" (Justicia, Benítez, Fernández-Cabezas, Fernández, y Pichardo-Martínez, 2008) que ha mostrado su eficacia en la mejora de la competencia social en alumnos de educación infantil (Alba, FernándezCabezas, Justicia, y Pichardo, 2015; Benítez, Fernández, Justicia, Fernández, y Justicia, 2011); el 
programa Ulises para el aprendizaje y desarrollo del autocontrol emocional (Comas, Moreno, y Moreno, 2002); y el programa "Ser Persona y Relacionarse" (Morales, 2002).

De otro lado, desde el modelo de habilidad, el programa INTEMO (Ruiz-Aranda, Cabello, Salguero, Extremera, y Fernández-Berrocal, 2013) tiene como objetivo el desarrollo de la inteligencia emocional en adolescentes entre 12 y 18 años en ámbito educativo. El protocolo está dividido en 4 fases en las que se planean actividades para el desarrollo las 4 habilidades emocionales indicadas en el modelo de Salovey y Mayer (1990): (a) percepción emocional, (b) facilitación emocional, (c) comprensión emocional y (d) regulación emocional. Consta de 12 sesiones en las que se realizan actividades tanto en el aula como tareas para el hogar. El programa ha demostrado su eficacia en la reducción de aspectos emocionales de las conductas agresivas y promoción de las habilidades de empatía en adolescentes (Castillo, Salguero, Fernández-Berrocal, y Balluerka, 2013), así como en la promoción de la salud mental en adolescentes incluso a largo plazo (Ruiz-Aranda et al., 2012a) y ha mostrado efectos moderados en ajuste psicológico (Ruiz-Aranda, Salguero, Cabello, Palomera, y Fernández-Berrocal, 2012b).

En suma, la educación emocional debe, entre otras cosas, facilitar la integración del individuo en la sociedad. Esto, a su vez, requiere un ajuste constante y, por tanto, flexibilidad para hacer frente a las demandas que surgen cotidianamente. Las competencias emocionales son una herramienta útil para facilitar un ajuste emocional (Guil y Gil-Olarte, 2007) y optimizar el desarrollo humano; dando lugar a la prevención de diferentes comportamientos desadaptativos (Bisquerra, 2003).

Desde una perspectiva contextual se entiende la flexibilidad psicológica como "la habilidad de estar en contacto con el presente plena y conscientemente, cambiando o persistiendo en su comportamiento cuando este se dirige hacia sus valores" en lugar de intentar suprimir, modificar o cambiar la frecuencia o intensidad de las emociones y pensamientos o comportamientos (Greco, Lambert, y Baer, 2008). En este sentido, las terapias contextuales o de tercera generación tienen como objetivo aportar un repertorio conductual flexible en cuanto a su interacción con los pensamientos, emociones o sensaciones (en adelante, eventos privados), y tienen como foco de intervención común el análisis funcional de la conducta y la aceptación (Hayes, Villatte, Levin, y Hildebrandt, 2011). Entre estas, encontramos las terapias basadas en Mindfulness (Baer, 2003), que se caracterizan por una intervención centrada en la observación de los eventos privados y los estímulos ambientales con conciencia y atención plena, sin jugarlos, con aceptación y compasión. Koole (2009) señala puntos comunes entre la IE y las habilidades desarrolladas a través del mindfulness. De hecho, la percepción y regulación emocional comparten aspectos con la consciencia que se tiene sobre los eventos privados de uno mismo y la reacción ante tales eventos privados en particular, de manera que el entrenamiento en mindfulness facilita la comprensión y regulación emocional (Baer, Smith y Allen, 2004; Bao, Xue, y Kong, 2015; Schutte y Malouff, 2011). En este sentido, el programa Aulas Felices (Arguís, Bolsas, Hernández, y Salvador, 2010), tiene como objetivo el fomento del desarrollo socio-emocional de adolescentes a través de componentes de mindfulness y de fortaleza psicológicas.

El presente estudio tiene como objetivo explorar los efectos de dos intervenciones para el desarrollo de competencias emocionales, una de ellas aplicada a un grupo de estudiantes y basada en el programa INTEMO (Ruiz-Aranda et al., 2013) frente a una segunda intervención en otro grupo con un protocolo de mindfulness para el fomento de habilidades de observación 
consciente y de toma de perspectiva. Así como valorar las habilidades y competencias que han influido en el desarrollo de la IE para cada uno de las intervenciones.

\section{Método}

\section{Participantes}

La muestra total está compuesta por 215 alumnos con edades comprendidas entre 12 y 15 años $(\mathrm{M}=12 \mathrm{DT}=.78)$ de primer y segundo curso de educación secundaria obligatoria de un centro escolar público. La muestra final, de carácter incidental, queda reducida a 120 de los alumnos de la muestra inicial, debido a pérdidas de participantes por absentismo escolar a lo largo de la intervención o en la toma de datos. Los criterios de exclusión fueron: la existencia de barreras idiomáticas o el diagnóstico de trastornos dentro del espectro autista o discapacidad intelectual.

El 54.2\% de la muestra total son varones. El 52.5\% $(n=63)$ de los alumnos conforman el grupo experimental 1, correspondiente al protocolo basado en el programa INTEMO de los cuales el 52.4\% son varones con una edad media de 12 años (DT=.66); mientras que el 47.5\% $(\mathrm{n}=57)$ pertenecían al grupo experimental 2 con un protocolo para fomentar la flexibilidad psicológica con ejercicios propios de mindfulness (en adelante protocolo mindfulness), cuyos participantes, el 56.1\% son varones, y tienen una edad media de 13 años (DT=.83).

El proceso de selección y delimitación de la muestra se resume en el diagrama de participantes (CONSORT) ilustrado en la Figura 2.

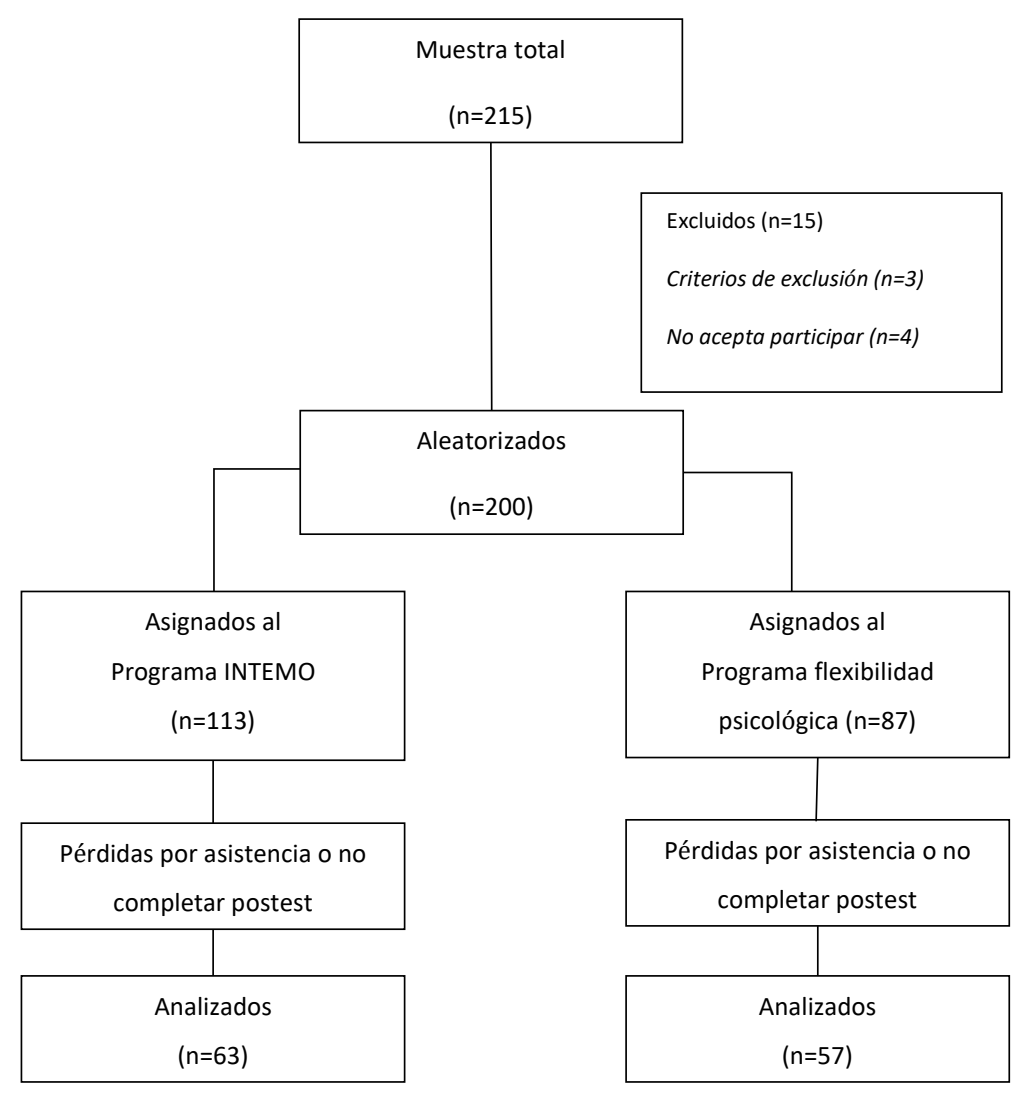

Figura 2. Diagrama de participantes (CONSORT) 


\section{Instrumentos}

Con el objetivo de medir los efectos en la percepción de las habilidades de inteligencia emocional se empleó el Trait Meta-Mood Scale (TMMS-24; Fernández-Berrocal, Extremera y Ramos, 2004). Consta de tres escalas: (a) atención hacia las emociones, (b) claridad emocional, y (c) reparación emocional. Los autores nombraron la puntuación global obtenida como Índice de Inteligencia Emocional Percibida (IEP). El cuestionario consta de 24 ítems de escala Likert de 5 puntos. Cuenta con buenas propiedades psicométricas con un alfa de Cronbach por encima de .85 y un coeficiente de estabilidad test-retest después de cuatro semanas de .60 para atención, .70 para claridad y .83 para reparación.

Para medir los efectos en las habilidades de mindfulness se utilizó el Cuestionario de Mindfulness de Cinco Factores (FFMQ; Cebolla, Garcia-Palacios, Soler, Guillen, Baños, y Botella, 2012) que mide la tendencia general a actuar con conciencia plena en la vida cotidiana. Consta de 39 ítems tipo Likert de 5 puntos. El FFMQ se compone de cinco componentes: observación, descripción, actuar con conciencia, ausencia de juicio, y ausencia de reactividad. El instrumento cuenta con buenas propiedades psicométricas en consistencia interna con valores entre .75 a .91 .

Como medida de la habilidad de regulación emocional desde el modelo de Gross y John (2003) se empleó el Cuestionario de Regulación Emocional (ERQ; Cabello, Ruiz-Aranda, Salguero y Castillo, 2009) que consta de 10 ítems con escala tipo Likert de 7 puntos, que conforman las subescalas de reevaluación cognitiva y de supresión emocional. El cuestionario muestra una consistencia interna con alfa de Cronbach de .79 para reevaluación cognitiva y de .73 para supresión emocional.

Finalmente, como otra de las competencias emocionales y de capacidad de toma de perspectiva y de comprensión de las emociones de los demás, se midió el Cociente de Empatía (EQ: Baron-Cohen y Wheelwright, 2004). El cuestionario consta de 60 ítems con escala Likert de 4 puntos, de las cuales 20 actúan como filtros; se obtiene una puntuación total llamada cociente de empatía. El cuestionario cuenta con una versión española del mismo autor. La fiabilidad del cuestionario es alta con una alfa de Cronbach de .92

\section{Procedimiento}

Se llevó a cabo el estudio con un diseño cuasi-experimental pre-post test de dos grupos independientes con medidas repetidas. Una primera condición se basa en el programa estandarizado INTEMO (Ruiz-Aranda et al., 2013) y una segunda condición consiste en un protocolo elaborado al efecto para el fomento de la flexibilidad psicológica a través de ejercicios propios de mindfulness. La intervención tuvo una duración de cinco sesiones semanales, con una hora de duración durante las horas de acción tutorial.

INTEMO es un programa de educación emocional orientado a jóvenes entre 12 y 18 años, basado en el modelo teórico de Salovey y Mayer (1990). El programa está estructurado en 4 fases de intervención orientadas al desarrollo de habilidades de: (a) percepción de las emociones en uno mismo y en los demás, (b) facilitación emocional para comprender las relaciones entre las emociones y las reacciones comportamentales, (c) comprensión de las emociones, y (d) regulación emocional a través de actividades dinámicas y de juego que se realizan en pequeños grupos dentro del aula. 
De otro lado, se elaboró un protocolo de comparación con el objetivo de fomentar habilidades de mindfulness. Concretamente, se realizaron ejercicios de comprensión y observación de las emociones, pensamientos y sensaciones corporales a través de estrategias de mindfulness para el fomento de una observación consciente y de la aceptación de estos eventos privados (Christensen, Riddoch, y Eggers Huber, 2009; García-Melón, 2009; Hanh, 2011; Harris, 2009). Posteriormente, se realizan ejercicios de toma de perspectiva o distanciamiento respecto a estos eventos privados. Estos objetivos se abordaron a través de ejercicios, actividades y metáforas que fomentasen la desliteralización de los eventos privados y se establecieron relaciones con aspectos personales que los participantes estaban viviendo y el contenido del ejercicio para fomentar la generalización.

En la Tabla 1 se ilustran los ejercicios de ambas intervenciones. Por un lado, los ejercicios del programa INTEMO fueron seleccionados por dos de las autoras con más de 20 años experiencia en intervención clínica y escolar bajo el criterio de mayor representatividad de las habilidades de IE. Por otro lado, el protocolo de mindfulness se desarrolló a partir de la consecución de los objetivos señalados anteriormente.

Tabla 1. Ejercicios empleados en los protocolos de intervención

\begin{tabular}{|c|c|c|}
\hline & Programa INTEMO & Protocolo de Mindfulness \\
\hline 1 & $\begin{array}{l}\text { FASE 1: Percepción y expresión de las emociones. } \\
\text { Qué dice tu cuerpo } \\
\text { Qué están sintiendo } \\
\text { Busca a tu par } \\
\text { Museo de cera }\end{array}$ & $\begin{array}{l}\text { Presentación teórica del Mindfulness } 1 \\
\text { Ejercicio de la uva pasa } \\
\text { Metáfora: La mente en un jarro } \\
\text { Primera práctica de meditación }\end{array}$ \\
\hline 2 & $\begin{array}{l}\text { FASE 2: Facilitación emocional. } \\
\text { Recuerdos emocionados } \\
\text { Cada emoción con su situación } \\
\text { Dime cómo te sientes y te diré cómo piensas }\end{array}$ & $\begin{array}{l}\text { Presentación teórica: Mindfulness } 2 \\
\text { Atención plena de la respiración } \\
\text { Ejercicio tratar de no pestañear } \\
\text { Ejercicio andar con consciencia }\end{array}$ \\
\hline 3 & $\begin{array}{l}\text { FASE 3: Comprensión de las emociones } \\
\text { Party (fiesta) de las emociones } \\
\text { El viaje en globo }\end{array}$ & $\begin{array}{l}\text { Atención plena de la mano } \\
\text { Ejercicio de las mariposas } \\
\text { Ejercicio de la mano no dominante } \\
\text { Entrenamiento a la meditación con las suelas de los pies }\end{array}$ \\
\hline 4 & $\begin{array}{l}\text { Pienso luego siento } \\
\text { Cambiando de rol. }\end{array}$ & $\begin{array}{l}\text { Ejercicio del dique } \\
\text { Aceptación de emociones } \\
\text { Ejercicio contar hasta } 10 \\
\text { Ejercicio de defusion (distanciamiento) } \\
\text { Metáfora el invitado incómodo en la fiesta }\end{array}$ \\
\hline 5 & $\begin{array}{l}\text { FASE 4: Regulación emocional } \\
\text { Qué hacemos cuando nos sentimos... } \\
\text { Qué me hacen sentir } \\
\text { Sabemos manejar las emociones de los demás }\end{array}$ & $\begin{array}{l}\text { Ejercicio de los sobres } \\
\text { Metáfora de las arenas movedizas } \\
\text { Metáfora de la Tortuga } \\
\text { Ejercicios de defusion-distanciamiento- } \\
\text { Metáfora ya sabes conducir }\end{array}$ \\
\hline
\end{tabular}




\section{Análisis estadístico}

Se llevó a cabo el análisis estadístico de los datos a través del programa estadístico IBM SPSS Statistics 21.0. para Mac. En primer lugar, se obtuvieron estadísticos descriptivos (media y desviación típica). Posteriormente, se realizó una prueba $T$ para muestras independientes a fin de comprobar si existían diferencias significativas en las puntuaciones pretest entre los grupos de intervención. Se analizaron las diferencias en las medias pre-postest a través de la prueba $T$ de Student para muestras relacionadas para cada uno de los grupos de intervención a fin de comprobar los efectos de las intervenciones. Dado que se encontraron diferencias en las puntuaciones pretest entre los grupos, se realizaron diferentes análisis de covarianza (ANCOVA) con cada una de las variables del estudio, empleando la variable grupo como factor con dos niveles (grupo experimental 1: Programa INTEMO y grupo experimental 2: protocolo mindfulness), las puntuaciones de las variables en el postest como variables dependientes (VD) y las puntaciones de esas mismas variables en el pretest como covariables para controlar los efectos de las puntuaciones pre-test. Se analizó el tamaño del efecto con el índice $d$ de Cohen, para el que se considera un tamaño del efecto pequeño para una puntuación de .20 , mediano para $.50 \mathrm{y}$ grande para .80 (Cohen, 1988). Por último, para conocer qué variables predicen el desarrollo de la IE, se realizó un análisis de regresión múltiple tipo stepwise en el que se toman las puntuaciones del postest en inteligencia emocional del TMMS-24 como variable dependiente y el resto de escalas como variables independientes para cada uno de los grupos experimentales.

\section{Resultados}

En la tabla 2 se recogen las medias y desviaciones típicas de las puntuaciones pretest y postest de cada uno de los grupos experimentales y el estadístico $T$ de Student y $F$ del ANCOVA realizado para cada una de las variables del estudio.

En primer lugar, para asegurar que la aleatorización fue efectiva, se compararon las puntuaciones en el pre-test en ambos grupos. Los resultados del análisis de las diferencias entre las medias de los grupos por medio de la prueba $T$ para muestras independientes mostró que no existían diferencias significativas en las variables, excepto para la supresión emocional ( $T=$ $2.012(118$ g.l. $) p=.046)$ y actuar con conciencia $(T=-2.152(118$ g.l. $) p=.035)$.

En el análisis de los efectos de la intervención a través de la prueba $T$ de Student para muestras relacionadas, en el programa INTEMO se encuentra que aumentan las puntuaciones con diferencias estadísticamente significativas en: atención emocional ( $T=3.18$ (62 g.l.) $p=.003)$, claridad emocional $(T=2.54$ (62 g.l.) $p=.013)$, reparación emocional $(T=2.904$ (62 g.l.) $p=.005)$, IEP $(T=4.015$ (62 g.l.) $p=0)$, empatía $(T=3.473(62$ g.l. $) p=.001)$ y observación $(T=3.888$ (62 g.l.) $p=.0)$. En el protocolo de mindfulness se encuentra que aumentan las puntuaciones con diferencias estadísticamente significativas en: atención emocional $(T=2.975$ (56 g.l.) $p=.004)$, claridad emocional $(T=5.609$ (56 g.l.) $p=0)$, reparación emocional $(T=6.51$ (56 g.l.) $p=0)$, IEP $(T=6.394$ (57 g.l.) $p=0)$, empatía $(T=2.563$ (56 g.l.) $p=.013)$ y observación $(T=2.919$ (57 g.l.) $p=.005)$, mientras que disminuye la puntuación de manera estadísticamente significativa en supresión emocional $(T=2.9(56$ g.l. $) p=.005)$, (véase Tabla 2$)$.

En cuanto al análisis de $A N C O V A$ realizados para evaluar las diferencias entre grupos, después de haber controlado las medias pretest, en el grupo de mindfulness se han hallado puntuaciones inferiormente significativas frente al grupo INTEMO en supresión emocional 
$(F=4.526, p<.05)$; mientras que el grupo INTEMO obtiene puntuaciones inferiormente significativas en reparación emocional $(F=5.096, p<.05)$ e IEP $(F=4.357, p<.05)$ frente al grupo de mindfulness (véase Tabla 2). Además, se calculó el tamaño del efecto empleando la $d$ de Cohen para las que se obtuvieron tamaños de efecto pequeños para supresión emocional $(\mathrm{d}=.19)$, reparación emocional $(d=.20)$ e IEP $(d=.19)$

Tabla 2. Puntuaciones pretest y postest (media y desviación típica), efectos de la intervención con t de Student y diferencias entre los grupos mediante ANCOVA

\begin{tabular}{|c|c|c|c|c|c|c|c|c|c|c|}
\hline & \multicolumn{4}{|c|}{ Programa INTEMO $(n=63)$} & \multicolumn{4}{|c|}{ Protocolo Mindfulness $(n=58)$} & \multicolumn{2}{|c|}{$A N C O V A$} \\
\hline & Pre & Post & $t$ & $p$ & Pre & Post & $t$ & $p$ & $\boldsymbol{F}$ & $p$ \\
\hline $\begin{array}{l}\text { Atención } \\
\text { emocional }\end{array}$ & $\begin{array}{l}22.87 \\
(5.16)\end{array}$ & $\begin{array}{l}25.14 \\
(5.58)\end{array}$ & 3.128 & $.003 *$ & $\begin{array}{l}22.05 \\
(6.61)\end{array}$ & $\begin{array}{l}25.04 \\
(7.48)\end{array}$ & 2.975 & $.004 *$ & .635 & .427 \\
\hline $\begin{array}{l}\text { Claridad } \\
\text { emocional }\end{array}$ & $\begin{array}{l}25.13 \\
(7.04)\end{array}$ & $\begin{array}{l}27.46 \\
(5.18)\end{array}$ & 2.549 & $.013 *$ & $\begin{array}{l}23.67 \\
(6.41)\end{array}$ & $\begin{array}{l}28.39 \\
(5.94)\end{array}$ & 5.609 & $0^{*}$ & 2.772 & .099 \\
\hline $\begin{array}{l}\text { Reparación } \\
\text { emocional }\end{array}$ & $\begin{array}{l}25.76 \\
(6.41)\end{array}$ & $\begin{array}{l}28.29 \\
(6.71)\end{array}$ & 2.904 & $.005^{*}$ & $\begin{array}{l}23.89 \\
(6.34)\end{array}$ & $\begin{array}{l}29.21 \\
(6.56)\end{array}$ & 6.51 & $0 *$ & 5.094 & $.026^{*}$ \\
\hline IEP & $\begin{array}{l}73.76 \\
(13.7)\end{array}$ & $\begin{array}{c}80.88 \\
(11.62)\end{array}$ & 4.015 & $0^{*}$ & $\begin{array}{c}69.61 \\
(15.11)\end{array}$ & $\begin{array}{c}82.63 \\
(14.63)\end{array}$ & 6.394 & $0 *$ & 4.357 & $.039 *$ \\
\hline Observación & $\begin{array}{l}24.41 \\
(5.79)\end{array}$ & $\begin{array}{l}27.02 \\
(5.68)\end{array}$ & 3.888 & $0^{*}$ & $\begin{array}{l}23.67 \\
(5.24)\end{array}$ & $\begin{array}{l}26.63 \\
(6.16)\end{array}$ & 2.919 & $.005 *$ & .424 & .516 \\
\hline Descripción & $\begin{array}{l}25.11 \\
(4.07)\end{array}$ & $\begin{array}{l}25.75 \\
(4.55)\end{array}$ & 1.128 & .264 & $\begin{array}{l}24.96 \\
(7.70)\end{array}$ & $\begin{array}{l}24.47 \\
(4.11)\end{array}$ & -.484 & .63 & .169 & .681 \\
\hline $\begin{array}{l}\text { Actuar con } \\
\text { conciencia }\end{array}$ & $\begin{array}{l}25.71 \\
(4.75)\end{array}$ & $\begin{array}{l}25.62 \\
(4.85)\end{array}$ & -.163 & .871 & $\begin{array}{l}24.68 \\
(4.14)\end{array}$ & $\begin{array}{l}23.6 \\
(5.44)\end{array}$ & 1.527 & .123 & .082 & .775 \\
\hline $\begin{array}{l}\text { Ausencia de } \\
\text { juicio }\end{array}$ & $\begin{array}{l}25.52 \\
(5.19)\end{array}$ & $\begin{array}{l}24.92 \\
(5.19)\end{array}$ & -.953 & .344 & $\begin{array}{l}26.44 \\
(5.69)\end{array}$ & $\begin{array}{l}25.67 \\
(5.67)\end{array}$ & -.919 & .362 & .419 & .519 \\
\hline $\begin{array}{l}\text { Ausencia de } \\
\text { reactividad }\end{array}$ & $\begin{array}{l}21.14 \\
(3.45)\end{array}$ & $\begin{array}{l}21.05 \\
(5.19)\end{array}$ & -.146 & .885 & $\begin{array}{l}20.09 \\
(3.79)\end{array}$ & $\begin{array}{l}20.46 \\
(4.44)\end{array}$ & .66 & .512 & 2.05 & .155 \\
\hline FFMQ Total & $\begin{array}{l}121.9 \\
(11.8)\end{array}$ & $\begin{array}{c}124.3 \\
(12.03)\end{array}$ & 1.757 & .084 & $\begin{array}{l}119.84 \\
(13.8)\end{array}$ & $\begin{array}{l}120.82 \\
(11.03)\end{array}$ & .475 & .637 & .05 & .824 \\
\hline Reevaluación & $\begin{array}{c}19.44 \\
(12)\end{array}$ & $\begin{array}{l}18.76 \\
(11.5)\end{array}$ & -.927 & .357 & $\begin{array}{c}16.01 \\
(10.90)\end{array}$ & $\begin{array}{l}15.60 \\
(9.87)\end{array}$ & -.573 & .569 & .211 & .647 \\
\hline Supresión & $\begin{array}{l}11.17 \\
(6.61)\end{array}$ & $\begin{array}{l}11.57 \\
(6.89)\end{array}$ & .724 & .472 & $\begin{array}{l}11.13 \\
(6.55)\end{array}$ & $\begin{array}{c}9.25 \\
(5.62)\end{array}$ & -2.9 & $.005 *$ & 4.526 & $.035^{*}$ \\
\hline Empatía & $\begin{array}{l}48.33 \\
(12.7)\end{array}$ & $\begin{array}{c}51.6 \\
(12.85)\end{array}$ & 3.473 & $.001^{*}$ & $\begin{array}{c}46.26 \\
(13.76)\end{array}$ & $\begin{array}{c}49.26 \\
(14.68)\end{array}$ & 2.563 & $0.013^{*}$ & .026 & .871 \\
\hline
\end{tabular}

$* p<.05$ 
Finalmente, se realiza un análisis de regresión múltiple paso a paso (stepwise) en el que se incluyó como variables dependientes la puntuación postest total de inteligencia emocional del TMMS-24 y como variable independiente las subescalas de los instrumentos, es decir, del ERQ, del EQ y del FFMQ, para cada uno de los grupos o protocolos. En la tabla 3 se ofrecen los resultados del análisis en el que encontramos que para el programa INTEMO el modelo que explica la mayor parte de la variabilidad de la inteligencia emocional $\left(R^{2}=41.8\right)$ incluye las variables de reevaluación cognitiva del ERQ $(\beta=.542)$ y descripción del FFMQ $(\beta=.438)$, en ambos casos la relación entre variables es positiva, la variable que mayor importancia adquiere en el modelo es la reevaluación cognitiva. Este segundo modelo de regresión aumenta en un $18.8 \%$ la explicación de un primer modelo de regresión en el que sólo incluye la variable reevaluación cognitiva $(\beta=.480)$ como predictor. Mientras que en el protocolo de mindfulness la variable observación consciente del FFMQ explica la variabilidad en relación a la inteligencia emocional de manera positiva $(\beta=.464)$, que puede explicar el $21.5 \%\left(R^{2}=21.5\right)$ de la variabilidad de la inteligencia emocional.

Tabla 3. Análisis de Regresión Múltiple Stepwise

\begin{tabular}{|c|c|c|c|c|c|c|}
\hline & & \multicolumn{2}{|c|}{$\begin{array}{l}\text { Coeficientes no } \\
\text { estandarizados }\end{array}$} & \multirow{2}{*}{$\begin{array}{c}\text { C. } \\
\text { Estandarizados } \\
\text { Beta }\end{array}$} & \multirow[t]{2}{*}{$t$} & \multirow[t]{2}{*}{ Sig. } \\
\hline & & $\boldsymbol{B}$ & Error típ. & & & \\
\hline \multicolumn{7}{|c|}{ Programa INTEMO } \\
\hline \multirow[t]{2}{*}{1} & (Constante) & 63.033 & 2.941 & & 21.436 & .000 \\
\hline & Reevaluación & .552 & .129 & .480 & 4.278 & .000 \\
\hline \multirow[t]{4}{*}{2} & (Constante) & 24.424 & 9.146 & & 2.671 & .010 \\
\hline & Reevaluación & .623 & .114 & .542 & 5.453 & .000 \\
\hline & Descripción & 1.482 & .337 & .438 & 4.400 & .000 \\
\hline & \multicolumn{6}{|c|}{ Protocolo de Mindfulness } \\
\hline 1 & (Constante) & 37.995 & 8.345 & & 4.553 & .000 \\
\hline & Observación & 1.336 & .344 & .464 & 3.879 & .000 \\
\hline
\end{tabular}

\section{Discusión}

El objetivo del presente trabajo es explorar la eficacia de dos programas de intervención para el desarrollo de la IE o competencias emocionales, uno basado en el programa INTEMO y otro elaborado al efecto con estrategias de mindfulness. Así como, conocer qué variables han podido influir en estos cambios.

En el análisis de los efectos de la intervención encontramos que el programa INTEMO obtiene diferencias estadísticamente significativas en las habilidades de prestar atención, comprender y regular sus emociones, así como en la IEP; resultado que es consecuente con estudios anteriores en los que la aplicación del programa INTEMO ha tenido efectos sobre las habilidades de IE (Castillo et al., 2013; Ruiz-Aranda et al., 2012a). Además, se ha mostrado un cambio significativo en empatía y observación consciente de las emociones. 
Por otro lado, el protocolo de mindfulness ha reducido significativamente la puntuación en estrategias de regulación emocional basadas en la supresión emocional. Además, ha obtenido cambios significativos en las puntuaciones de atención, claridad y reparación emocional, IEP, empatía y observación consciente de las emociones.

En la comparación de puntuaciones entre grupos, se han encontrado diferencias significativas en supresión emocional, reparación emocional e IEP. Concretamente, el protocolo de mindfulness ha obtenido puntuaciones mayores en IEP y reparación emocional, mientras que ha reducido en mayor medida la supresión emocional al finalizar la intervención en comparación con el programa INTEMO.

Finalmente, se han hallado como predictores de la variabilidad de la inteligencia emocional las variables de reevaluación cognitiva y descripción de emociones para el programa INTEMO, estos resultados son coherentes con el objetivo de la intervención ya que entre las estrategias de regulación emocional tienen a la base estrategias de carácter cognitivoconductual como son las técnicas percepción emocional y de reestructuración cognitiva.

Sin embargo, en el protocolo de mindfulness la variable predictora de la inteligencia emocional es la observación, esto es, la capacidad para observar de manera consciente y abierta las experiencias internas y externas (Baer et al., 2006), entre las que se encuentran las emociones, sin juzgarlas. La influencia de la observación consciente de las emociones en la inteligencia emocional ha sido explorada por otros autores (Baer et al., 2004; Baer et al., 2006; Koole, 2009), así como también se han relacionado las habilidades de mindfulness con el desarrollo de IE (Bao et al., 2015; Charoensukmongkol, 2014; Schutte y Malouff, 2011; Wang y Kong, 2014). Es destacable que la mayoría de los ejercicios realizados a lo largo del protocolo de mindfulness están orientados a la defusion (o distanciamiento) cognitiva y observación con atención plena de las emociones, sensaciones y pensamientos en diferentes contextos personales, por lo que la influencia de esta habilidad en la inteligencia emocional queda demostrada para la muestra del estudio. No obstante, esta focalización en ejercicios de observación consciente, puede explicar la ausencia de efectos del resto de habilidades de mindfulness evaluadas, lo que señala la necesidad de ampliar el espectro de ejercicios para potenciar el resto de habilidades como: la acción consciente y con significado, descripción y etiquetado de las emociones sin juzgar y con aceptación.

A la luz de estos resultados, podemos concluir que tanto el programa INTEMO como el protocolo de mindfulness producen cambios significativos en IE y en sus habilidades como son la atención, comprensión y regulación emocional, es decir, ambos protocolos muestran eficiencia en el incremento de habilidades de inteligencia emocional. No obstante, las estrategias de regulación de estas emociones son diferentes.

Por un lado, los resultados del programa INTEMO muestran una mayor tendencia a emplear estrategias de modulación de respuesta y cambio cognitivo desde el modelo de regulación emocional de Gross (1998). Concretamente, los adolescentes que han recibido intervención siguiendo el programa INTEMO, obtienen puntuaciones mayores en supresión, esto se refiere a los intentos por cambiar la apariencia, reacciones o conductas producidas por la emoción. Además, como variable predictora de las puntuaciones se encuentra la reevaluación, esto es, cambiar cognitivamente la interpretación o el significado emocional de una situación de manera que cambie la respuesta que produce. 
Por otro lado, los resultados del protocolo de mindfulness muestran que los adolescentes obtienen mayor puntuación en reparación emocional al finalizar la intervención en comparación con el programa INTEMO, esto es, la habilidad para regular las emociones negativas hacia un pensamiento positivo desde el modelo de habilidad (Salovey, Stroud, Woolery, \& Epel, 2002). Asimismo, en cuanto a las variables predictoras encontramos que la observación consciente de las emociones predice los resultados de esta intervención. Estudios anteriores muestran que las habilidades en mindfulness e inteligencia emocional ayudan a la percepción y comprensión emocional de uno mismo y de los demás y, por ende, a mejorar la regulación emocional (Brown, Ryan y Creswell, 2007).

Por tanto, en un análisis de las implicaciones clínicas de ambas intervenciones, podemos concluir que ambos protocolos mejoran la IE, pero que las estrategias de regulación son diferentes. La intervención con INTEMO promueve estrategias de cambio cognitivo orientadas a la evitación o supresión emocional, mientas que el protocolo de mindfulness tiende a observar conscientemente estas emociones y orientarlos a una reevaluación positiva. Diversos estudios señalan que las estrategias de control emocional, como la supresión de emociones o la evitación, conllevan efectos paradójicos a largo plazo, aunque puedan parecer efectivas a corto plazo (Campbell-Sills, Barlow, Brown, y Hofmann, 2006; Geraerts, Merckelbach, Jelicic, \& Smeets, 2006). Por ello, sería recomendable que las intervenciones para el fomento de las competencias emocionales promuevan estas estrategias de regulación emocional adaptativas (Aldao, Nolen-Hoeksema, \& Scheweizer ,2010) como sucede en el protocolo de mindfulness.

Como limitaciones del estudio se encuentran principalmente: el no haber usado un grupo control sin intervención, un tamaño de muestra reducido que puede debilitar la generalización de los resultados, y el uso de medidas autoinformadas. Por ello, sería conveniente replicar este estudio ampliando la muestra y las fuentes de información (por ejemplo, añadir información dada por profesores y/o familiares), así como un seguimiento a largo plazo. A su vez, cabe señalar la necesidad de elaborar, en futuros estudios, un protocolo que amplíe los componentes de mindfulness más orientados a la acción y que sea capaz de generalizarse a la historia personal en su aplicación en grupo para adolescentes y que contemple una batería de evaluación orientada a estrategias específicas de regulación emocional, como los planteados por Seligowski y Orcutt (2015).

En resumen, este estudio ha añadido evidencia empírica a la aplicación de los programas de desarrollo de percepción, compresión y regulación de las emociones en contextos escolares en la adolescencia, incorporando un protocolo para el fomento de habilidades de mindfulness que ha producido cambios significativos en las habilidades de inteligencia emocional. Asimismo, se han hallado factores que parecen estar a la base del desarrollo de inteligencia emocional y mindfulness, encontrando cierta conexión entre ambas.

\section{Referencias}

Alba, G., Fernández-Cabezas, M., Justicia, F., y Pichardo, M. C. (2015). The longitudinal effect of the aprender a convivir (learning to live together) programme in childhood: The development of social competence. Cultura Y Educación, 27(1), 186-220. DOI:10.1080/11356405.2015.1012804

Aldao, A., Nolen-Hoeksema, S., \& Schweizer, S. (2010). Emotion-regulation strategies across psychopathology: A meta-analytic review. Clinical Psychology Review, 30, 217-237. https://doi.org/10.1016/j.cpr.2009.11.004 
Arguís, R., Bolsas, A. P., Hernández, S., y Salvador, M. (2010). Programa “Aulas felices”. psicología positiva aplicada a la educación. Zaragoza.SATI. Recuperado de http://catedu.es/psicologiapositiva/Aulas\%20felices.pdf

Baer, R. A. (2003). Mindfulness training as a clinical intervention: A conceptual and empirical review. Clinical Psychology: Science and Practice, 10(2), 125-143. https://doi.org/10.1093/clipsy/bpg015

Baer, R. A., Smith, G. T., y Allen, K. B. (2004). Assessment of mindfulness by self-report: The kentucky inventory of mindfulness skills. Assessment, 11(3), 191-206. Recuperado de http://www.ncbi.nlm.nih.gov/pubmed/15358875

Bao, X., Xue, S., y Kong, F. (2015). Dispositional mindfulness and perceived stress: The role of emotional intelligence. Personality and Individual Differences, 78, 48-52. doi: 10.1016/j.paid.2015.01.007

Baron-Cohen, S., y Hammer, J. (1997). Parents of children with asperger syndrome: What is the cognitive phenotype? Journal of Cognitive Neuroscience, 9(4), 548-554. doi:10.1162/jocn.1997.9.4.548

Baron-Cohen, S., y Wheelwright, S. (2004). The empathy quotient: An investigation of adults with asperger syndrome or high functioning autism, and normal sex differences. Journal of Autism and Developmental Disorders, 34(2), 163-175. Recuperado de http://isik.zrcsazu.si/doc2009/kpms/Baron-Cohen_empathy_quotient_2004.pdf

Benítez, J. L., Fernández, M., Justicia, F., Fernández, E., y Justicia, A. (2011). Results of the aprender a convivir program for development of social competence and prevention of antisocial behavior in four-year-old children. School Psychology International, 32(1), 3-19. doi: $10.1177 / 0143034310396804$

Bisquerra, R. (2003). Educación emocional y competencias básicas para la vida. Revista De Investigación Educativa, 21(1), 7-43. Recuperado de http://revistas.um.es/rie/article/view/99071

Brown, K. W., Ryan, R. M., \& Creswell, J. D. (2007). Mindfulness: Theoretical foundations and evidence for its salutary effects. Psychological Inquiry, 18(4), 211-237. DOI:10.1080/10478400701598298

Cabello, R., Ruiz-Aranda, D., Salguero, J., y Castillo, R. (2009). Propiedades psicométricas del cuestionario ERQ de regulación emocional en una muestra española. En Fernández-Berrocal, P., Extremera, N., Palomera, R., Ruiz-Aranda, D., Salguero, J.M., Cabello, R. (Ed.), Avances en el estudio de la inteligencia emocional (pp. 15). Santander: Fundación Marcelino Botín.

Campbell-Sills, L., Barlow, D. H., Brown, T. A., \& Hofmann, S. G. (2006). Effects of suppression and acceptance on emotional responses of individuals with anxiety and mood disorders. Behaviour Research and Therapy, 44(9), 1251-1263. https://doi.org/10.1016/j.brat.2005.10.001

Carrasco, C., Alarcón, R., y Trianes, M. V. (2015). Effectiveness of an intervention programme based on social climate, school violence and sociometric tests in primary education. Journal of Psychodidactics, 20(2), 247-262 DOI: 10.1387/RevPsicodidact.13206

Castillo, R., Salguero, J. M., Fernández-Berrocal, P., y Balluerka, N. (2013). Effects of an emotional intelligence intervention on aggression and empathy among adolescents. Journal of Adolescence, 36(5), 883-892. Doi: 10.1016/j.adolescence.2013.07.001

Cebolla, A., Garcia-Palacios, A., Soler, J., Guillen, V., Baños, R., y Botella, C. (2012). Psychometric properties of the spanish validation of the five facets of mindfulness questionnaire (FFMQ). The European Journal of Psychiatry, 26(2), 118-126. Recuperado de http://scielo.isciii.es/scielo.php?script=sci_arttext\&pid=S0213-61632012000200005

Charoensukmongkol, P. (2014). Benefits of mindfulness meditation on emotional intelligence, general self-efficacy, and perceived stress: Evidence from thailand. Journal of Spirituality in Mental Health, 16(3), 171-192. DOI:10.1080/19349637.2014.925364 
Christensen, R., y Christensen, K. (2009). Dialectical Behavior Therapy Skills, 101 Mindfulness Exercises and Other Fun Activities for Children and Adolescents: A Learning Supplement. Bloomington: AuthorHouse.

Cobos, L., Flujas-Contreras, J.M., Gómez, I. (2015). Educación emocional como prevención y manejo de los trastornos de conducta. Conferencia llevada a cabo en el XXII Congreso Español de Pediatría Social en Almería.

Cohen, J. (1988). Statistical power analysis for the behavioral sciences. (Second, Ed.). Hillsdale, New Jersey: Lawrence Erlbaum Associates

Comas, R., Moreno, G., y Moreno, J. (2002). Programa Ulises: Aprendizaje y desarrollo del autocontrol emocional. Madrid: Asociación deporte y vida.

Fernández-Berrocal, P., Extremera, N., y Ramos, N. (2004). Validity and reliability of the spanish modifed version of the trait meta mood scale. Psychological Reports, 94(3), 751-755. Recuperado de http://emotional.intelligence.uma.es/pdfs/spanish\%20tmms.pdf

García-Fernández, M., \& Giménez-Mas, S. I. (2010). La inteligencia emocional y sus principales modelos: propuesta de un modelo integrador. Espiral. Cuadernos del profesorado, 3(6), 43-52.

García-Melón, M. (2009). Técnicas de psicoterapia con base en atención plena. Material no publicado. Recuperado a 9 de abril de 2014 en http://es.scribd.com/doc/217476930/14-1-Tecnicas-dePsicoterapia-en-Base-a- La-Atencion-Plena

Geraerts, E., Merckelbach, H., Jelicic, M., \& Smeets, E. (2006). Long term consequences of suppression of intrusive anxious thoughts and repressive coping. Behaviour Research and Therapy, 44(10), 1451-1460. https://doi.org/10.1016/J.BRAT.2005.11.001

Greco, L. A., Lambert, W., \& Baer, R. A. (2008). Psychological inflexibility in childhood and adolescence: Development and evaluation of the Avoidance and Fusion Questionnaire for Youth. Psychological Assessment, 20(2), 93-102. https://doi.org/10.1037/1040-3590.20.2.93

Gross, J. J., y John, O. P. (2003). Individual differences in two emotion regulation processes: Implications for affect, relationships, and well-being. Journal of Personality and Social Psychology, 85(2), 348. Recuperado de http://www.ncbi.nlm.nih.gov/pubmed/12916575

Gross, J. J. (1998). The emerging field of emotion regulation: An integrative review. Review of General Psychology, 2, 271-299. https://doi.org/10.1037/1089-2680.2.3.271

Guil, R., y Gil-Olarte, P. (2007). Inteligencia emocional y educación: Desarrollo de competencias socioemocionales. In J. M. Mestre, \& P. Fernández (Eds.), Manual de inteligencia emocional (pp. 187). Madrid: Ediciones Pirámide.

Hanh, T. N. (2011). Planting seeds: Practicing mindfulness with children. Berkeley, CA; Parallax Press.

Harris, R. (2009). ACT made simple: An easy-to-read primer on acceptance and commitment therapy. Oakland, CA: New Harbinger Publications.

Hayes, S. C., Villatte, M., Levin, M., \& Hildebrandt, M. (2011). Open, Aware, and Active: Contextual Approaches as an Emerging Trend in the Behavioral and Cognitive Therapies. Annual Review of Clinical Psychology, 7(1), 141-168. https://doi.org/10.1146/annurev-clinpsy-032210-104449

Justicia, F. J., Benítez, J. L., Fernández-Cabezas, M., Fernández, E., y Pichardo-Martínez, M.C. (2008). Aprender a convivir: Programas de prevención de comportamento antisocial na educación infantil. Cadernos de Psicoloxía, 32, 37-47. Recuperado de https://dialnet.unirioja.es/servlet/articulo?codigo $=2971261$

Koole, S. L. (2009). The psychology of emotion regulation: An integrative review. Cognition and Emotion, 23(1), 4-41. DOI:10.1080/02699930802619031

Mayer, J. D., Salovey, P., Caruso, D. R., y Sitarenios, G. (2001). Emotional intelligence as a standard intelligence. Emotion, 1(3). 232-242. DOI:10.1037//1528-3542.1.3.232

Morales, M. S. (2002). Ser persona y relacionarse: Habilidades cognitivas y sociales, y crecimiento moral. Madrid: Narcea Ediciones. 
Ruiz-Aranda, D., Castillo, R., Salguero, J. M., Cabello, R., Fernández-Berrocal, P., y Balluerka, N. (2012a). Short-and midterm effects of emotional intelligence training on adolescent mental health. Journal of Adolescent Health, 51(5), 462-467. doi: 10.1016/j.jadohealth.2012.02.003

Ruiz-Aranda, D., Salguero, J. M., Cabello, R., Palomera, R., y Fernández-Berrocal, P. (2012b). Can an emotional intelligence program improve adolescents' psychosocial adjustment? results from the intemo project. Social Behavior and Personality: An International Journal, 40(8), 1373-1379. http://dx.doi.org/10.2224/sbp.2012.40.8.13731373

Ruiz-Aranda, D., Cabello, R., Salguero, J. M., Extremera, N., y Fernández-Berrocal, P. (2013). Programa INTEMO. Guía para mejorar la inteligencia emocional de los adolescentes. Madrid: Pirámide.

Ruiz-Aranda, D., Fernández-Berrocal, P., Cabello, R., y Salguero, J. M. (2008). Educando la inteligencia emocional en el aula: Proyecto Intemo. Revista de Investigación Psicoeducativa, 6(2), 240-251. Recuperado de http://www.investigacion-psicopedagogica.org/revista/new/ContadorArticulo.php?256

Salovey, P., y Mayer, J. (1990). Emotional intelligence. Imagination, Cognitivon and Personality, 9, $185-212$.

Salovey, P., Stroud, L. R., Woolery, A., \& Epel, E. S. (2002). Perceived emotional intelligence, stress reactivity, and symptom reports: Further explorations using the trait meta-mood scale. Psychology and Health, 17(5), 611-627.

Schutte, N. S., y Malouff, J. M. (2011). Emotional intelligence mediates the relationship between mindfulness and subjective well-being. Personality and Individual Differences, 50(7), 11161119. doi: 10.1016/j.paid.2011.01.037

Seligowski, A. V., \& Orcutt, H. K. (2015). Examining the structure of emotion regulation: a factor-analytic approach. Journal of Clinical Psychology, 71, 1004-1022. https://doi.org/10.1002/jclp.22197

Trianes, M.V., y Fernández-Figares, C. (2001). Aprender a ser personas y a convivir. Bilbao: Descleé de Brouwer.

Trujillo Flores, M. M., \& Rivas Tovar, L. A. (2005). Orígenes, evolución y modelos de inteligencia emocional. Innovar, 15(25), 9-24.

Wang, Y., y Kong, F. (2014). The role of emotional intelligence in the impact of mindfulness on life satisfaction and mental distress. Social Indicators Research, 116(3), 843-852. Recuperado de http://link.springer.com/article/10.1007\%2Fs11205-013-0327-6 